\title{
Impact des appels à projets dans le secteur de l'aide à domicile
}

\author{
Marie-Claire Sepulchre \\ Dans Les PolitiQues Sociales 2015/1 (N 1-2), PAGeS 78 À 92 \\ ÉDITIONS SERVICE SOCIAL DANS LE MONDE
}

ISSN 1374-1942

Article disponible en ligne à l'adresse

https://www.cairn.info/revue-les-politiques-sociales-2015-1-page-78.htm

\author{
CAIRN I \\ MATIÈRES À RÉFLEXION
}

Découvrir le sommaire de ce numéro, suivre la revue par email, s'abonner...

Flashez ce QR Code pour accéder à la page de ce numéro sur Cairn.info.

Distribution électronique Cairn.info pour Service social dans le Monde.

La reproduction ou représentation de cet article, notamment par photocopie, n'est autorisée que dans les limites des conditions générales d'utilisation du site ou, le cas échéant, des conditions générales de la licence souscrite par votre établissement. Toute autre reproduction ou représentation, en tout ou partie, sous quelque forme et de quelque manière que ce soit, est interdite sauf accord préalable et écrit de l'éditeur, en dehors des cas prévus par la législation en vigueur en France. Il est précisé que son stockage dans une base de données est également interdit. 


\title{
Impact des appels à projets dans le secteur de l'aide à domicile
}

\section{Marie-Claire Sepulchre *}

* Fédération wallonne des services d'aide à domicile (FEDOM)

\begin{abstract}
Depuis le lancement des appels à projets, le secteur de l'aide à domicile s'est montré très en verve et a participé activement à ce nouveau mode de régulation et de gouvernance. Retraçant sept appels qui ont eu un impact prégnant sur la vie des services, nous nous proposons d'analyser les effets produits, à partir de la consultation des directions où la focale est mise sur les points évalués comme positifs par les services eux-mêmes.
\end{abstract}

\section{Introduction}

Nous commencerons par une rapide description du secteur de I'aide à domicile en Région Wallonne pour ensuite passer en revue les sept appels à projets sélectionnés. Enfin nous terminerons par une analyse transversale de ces projets. Notre étude de cas nous pousse à analyser ces projets en termes d'instruments des politiques publiques participant à une forme de professionnalisation du secteur en accentuant la division sociale et technique du travail. Créant des filières de spécialisation et de diversification des parcours dans l'optique du cadre européen de sécurisation des trajectoires professionnelles. L'organisation ainsi que les conditions de travail en sont profondément modifiées. De plus, nous nous arrêterons sur la question de la pérennisation du projet qui entraine une précarisation de l'em- 
ploi face aux incertitudes contractées. Enfin, nous aborderons une face souvent oublié des appels à projets, I'induction d'une réflexivité des services et la production d'innovation sociale en intégrant les usagers à la réflexion.

\section{Le secteur de l'aide à domicile en Wallonie}

Le secteur de l'aide à domicile en Wallonie est né à la fin des années 40 et a vu son importance croître à l'aune de la prise en charge de publics vulnérables tels les personnes âgées, handicapées, malades, ou des familles en difficulté. Le secteur s'articule autour d'une norme de services et de solidarité en permettant aux personnes de continuer à vivre chez elles tout en conservant un bien-être et une sécurité dans leur vie quotidienne. L'aide à domicile est non seulement importante pour le public directement aidé, mais le secteur soutient également les aidants proches et les familles, en permettant de maintenir le lien social entre les usagers et la société. Les services y opérant déploient une aide qui vise à apporter à ces personnes une solution adaptée et individualisée grâce à l'intervention régulière de professionnels de l'accompagnement: des aides familiales $(A F)$, des aides ménagères (AM), des gardes à domicile (GAD), des ouvriers polyvalents (OP)... encadrés par des travailleurs sociaux.

Elle prend la forme d'une aide régulière continue (parfois de très longue durée) et de proximité. La nature de cette aide est amenée à évoluer pour s'adapter aux demandes et besoins des usagers.

Le secteur de l'aide à domicile met donc en relation des usagers, des travailleurs et une entreprise de services, dont l'activité est règlementée et soutenue par l'Etat. Cette configuration triangulaire de la relation " prestataire-client-entreprise " est atypique, car le prestataire travaille en permanence au domicile du client, lieu d'intimité, alors qu'en général les travailleurs exercent leur activité professionnelle dans les locaux de l'entreprise. 
Cette réalité conditionne toute l'organisation de l'activité des services.

Etat

\begin{tabular}{|c|c|}
\hline \multicolumn{2}{|c|}{ Usager } \\
\hline Travailleur & Service \\
\hline
\end{tabular}

Par ailleurs, les services d'aide à domicile sont agréés et règlementés par les législations mises en œuvre par les régions ou communautés dont ils dépendent ${ }^{1}$. C'est un secteur qui repose sur un partenariat public-associatif mettant en présence des associations non lucratives publiques ou privées agréées et subventionnées par les pouvoirs publics. En Wallonie, l'agrément est attribué par la Direction Générale opérationnelle des Pouvoirs locaux, de l'action sociale et de la santé ${ }^{2}$ au titre de "Service d'aide aux familles et aux aînés " (SAFA). C'est un secteur d'activité stable et reconnu qui est en constante évolution, car les besoins de la population wallonne ne cessent de croître (vieillissement de la population, accroissement de la précarité, mise en œuvre de diverses politiques favorisant le maintien et le retour à domicile). Ces facteurs engendrent une croissance constante de l'emploi salarié au sein des services associatifs ${ }^{3}$.

Depuis la fin des années 90 , les services ont pu répondre à des appels à projets émanant des pouvoirs publics, de fondations, de coopératives ou d'associations. Certains services ont saisi l'opportunité de ces appels à projets avec des motivations diverses. Cet article vise à analyser les effets de ces projets sur la vie des entreprises qui les ont développés que ce soit à travers le mode de subsidiation, l'innovation de l'offre de services, les conditions de travail des salariés, ou encore l'adaptation de l'organisation du travail...

Dans cet article, nous nous limiterons à évoquer les appels à projets qui ont été développés dans certains SAFA. 


\section{Retour non exhaustif sur les types d'appel à projets développés dans le secteur de l'aide à domicile et leurs effets}

Selon notre conception, un appel à projets exprime le souhait d'une entité publique ou privée d'apporter son soutien à des projets relevant d'un secteur spécifique dans le but de répondre à un besoin par une nouvelle offre de service. Le soutien peut être financier, matériel ou technique. De ce fait, l'appel à projets a toujours une date limite de dépôt de dossiers et des critères de sélection définis dans un cahier des charges ${ }^{4}$. Nous allons dans cette partie nous intéresser à des appels à projets émanant du Gouvernement wallon, des Fondations CERA ou Roi Baudouin et, enfin, de la Province du Luxembourg

\subsection{Plan Marshall : aide aux personnes dépendantes}

Dans le cadre du plan Marshall ${ }^{5}$, le gouvernement wallon a voulu développer des pôles de compétitivité pour relancer l'activité économique de la Wallonie. Un pôle de développement était réservé au secteur non marchand et comportait 2 volets: le volet "Petite enfance " et le volet " Aide aux personnes dépendantes (âgées et/ou handicapées) ". Dans le cadre de ce $2^{\mathrm{e}}$ volet, certains gardes ont obtenu des postes de gardes à domicile et de gardes d'enfants malades (GEM) à domicile. Plus d'un millier d'emplois salariés a été octroyé à l'ensemble du secteur. Dans cet appel à projets, les emplois étaient financés par le ministre de l'Emploi des mesures d'aides à l'emploi ${ }^{6}$ et attribués par le ministre de l'Action sociale et de la Santé.

Missions des GAD et des GEM

Le garde à domicile a pour mission d'accompagner le bénéficiaire qui a besoin de la présence continue d'une personne et qui, pour des raisons de santé ou de handicap, ne peut se déplacer seul hors de son domicile. Il vise principalement à assurer, le jour et/ou la nuit, en complémentarité avec l'entourage du bénéficiaire, une présence active et à optimaliser le bien-être mental, physique et social du bénéficiaire.

Le garde d'enfant malade offre une aide aux parents d'enfants (de 0 à 12 ans), qui, pour des raisons médicales, doivent rester à la maison et ne peuvent fréquenter l'école, la crèche et /ou leur lieu habituel de garderie?.

Le métier de GAD existait de manière expérimentale dans certains services et souvent via des modes de financement limités (PTP) ou 
spécifiques (Maribel Social). La possibilité d'obtenir d'autres emplois via le plan Marshall a permis aux services, soit de consolider ce département d'activités, soit de créer une nouvelle offre de services à la population aidée. Cet appel à projets $\mathrm{n}^{\prime} a$ été éligible que pour les SAFA, certaines associations médicales et des associations s'occupant d'aide aux personnes handicapées. Malgré cela, nous n'avons pas remarqué de concurrence entre les services, car tous les services demandeurs ont obtenu des postes. Comme la demande des usagers était très importante, l'offre proposée n'a pas supprimé la présence du travail au noir. De plus, l'octroi des postes APE n'a été effectué qu'à durée déterminée. Chaque opérateur est donc tenu d'introduire régulièrement des demandes de renouvellement. C'est un élément d'insécurité et de précarisation d'emploi pour les opérateurs, les travailleurs ainsi que les usagers.

Au début du programme, ce métier n'était pas du tout réglementé. Depuis le décret de 2007 et l'arrêté de 2009, ce métier est pleinement entré dans les textes réglementaires des SAFA. Malgré tout, l'insertion de ce métier est incomplète, car il n'y a pas de subvention générale de la Région wallonne. La subvention n'est effectuée que via des programmes d'aide à l'emploi, la Région wallonne se contentant d'allouer quelques financements complémentaires. Seuls les emplois de prestataires sont pris en compte. Les services doivent s'organiser pour mettre en place les postes d'encadrement. Les métiers de GAD et GEM sont soutenus par des actions de formation continuée, mais les services n'obtiennent pas de financement complet de ces heures de formation.

La subvention APE est suffisante pour couvrir le coût du poste en début de carrière ${ }^{8}$ mais la prise en compte de l'ancienneté $n^{\prime}$ est pas effective. Ce métier n'est accessible qu'à des demandeurs d'emploi ayant un jour de chômage. Or beaucoup d'aides familiales expérimentées voudraient évoluer vers ce métier. Ces conditions d'embauche rendent la transition professionnelle AF/GAD impossible puisqu'un employeur ne peut engager en APE un travailleur qu'il a précédemment licencié.

Par ailleurs, il n'y a pas de barème de contributions des usagers fixé par la DGO5 pour le service de GAD. Chaque service est donc libre de fixer son prix ce qui détermine l'offre concurrentielle entre services, surtout que la facture de l'activité des GAD est conséquente 
pour les usagers.

Enfin, I'administration wallonne n'a hélas pas mis en œuvre un cadre règlementaire des visites d'inspection du département GAD. II $n^{\prime} y$ a pas d'autres rapports d'évaluation à fournir que celui demandé dans le cadre de la subvention APE.

\subsection{Projet répit pour les familles des personnes handicapées}

La garde répit est un service de garde à domicile qui soutient les familles ayant la prise en charge à domicile d'une personne handicapée reconnue par l'AWIPH. La garde répit leur permet de "souffler" et d'avoir un peu de temps "pour soi " pendant quelques heures ou quelques jours/nuits, de manière ponctuelle en prenant "le relais" auprès de la personne en situation de handicap. La garde répit assure une présence active auprès du bénéficiaire, en effectuant, d'une part, des tâches d'aide à la vie journalière et en remplissant, d'autre part, un rôle d'accompagnement relationnel et occupationnel. Cet appel à projets est soutenu par l'AWIPH et le Ministère de l'Emploi de la Région Wallonne (co-financement AWIPH/APE) et les travailleurs doivent donc correspondre aux conditions d'embauche APE.

Les SAFA ont une possibilité d'aider les personnes handicapées en fournissant des prestations d'AF. Toutefois, ce personnel travaille en horaire de jour et en horaire inconfortable $(6 \%$ du contingent $\mathrm{d}^{\prime}$ heures subventionnées) ${ }^{9}$. Les modalités d'organisation ne leur permettent pas d'effectuer des prestations importantes en soirée, la nuit, les weekends et les jours fériés. Ce projet a donc été une réelle opportunité de développer une offre de répit adaptée aux aidants proches vivant avec des personnes handicapées (adultes ou enfants), entre autres en soirée ou la nuit. Toutefois, le nombre limité de postes ne permet pas de répondre à l'ensemble des demandes des familles concernées.

Dès lors deux difficultés sont apparues: d'une part la lourdeur de gestion des horaires et d'autre part la limite des actes sanitaires autorisés pour les GAD qui ne permet pas de répondre à l'ensemble des demandes. En effet, les horaires des GAD sont complexes, car ils demandent de respecter la règlementation du travail sur divers aspects: horaire fixe ou horaire variable, travail de jour, travail en horaires inconfortables, travail de nuit, respect des périodes de repos... 
Comme il y a peu de postes, la couverture géographique du territoire est incomplète. II y a peu de possibilités de développer un travail d'équipe. Depuis le décret de 2007, les SAFA se sont vu confier une mission complémentaire d'aide aux aidants proches des bénéficiaires. L'arrêté de 2009 a très peu précisé cette nouvelle mission. L'expérience des services déployant une offre de répit a été une réelle opportunité pour réfléchir à leurs réalités et besoins spécifiques. $C^{\prime}$ est pourquoi, à partir de 2013, la règlementation sectorielle a permis à ces GAD de sortir du domicile avec les personnes handicapées. Cette possibilité de vivre ensemble des activités extérieures renforce la relation aux prestataires et permet ensuite de continuer des activités au domicile sur la même thématique. Les prestataires travaillant dans ce projet répit ont bénéficié de formations spécifiques à l'aide aux personnes handicapées et cela a eu un effet d'essaimage sur les autres gardes à domicile du service. Ce qui leur permet de gérer des situations très lourdes et complexes. Le service s'est ouvert sur la réalité des personnes handicapées, et a pu promotionner une image de souplesse, de polyvalence, de professionnalisme, de capacité d'adaptation et de compétences relationnelles. II a ainsi pu déployer une stratégie de diversification.

\subsection{Accueil familial des aînés}

Le projet accueil familial des aînés a été promu par la Ministre Tillieux pendant la législature 2009-2014. L'accueil familial des aînés est un projet pilote de la Région wallonne, qui s'adresse à des personnes âgées de plus de 60 ans qui sont peu ou pas dépendantes. II leur propose un nouveau mode d'hébergement, une solution alternative au maintien à domicile. Concrètement, un particulier accueille chez lui un aîné ou un couple d'aînés. La famille d'accueil et la ou les personne(s) accueillie(s) partagent le quotidien dans un cadre familial, chaleureux et sécurisant.

II s'agit de donner la possibilité aux aînés de continuer à vivre dans un environnement semblable à celui qu'ils ont toujours connu. L'accueil des aînés est un vrai choix de vie, créateur de liens privilégiés entre un aîné et un particulier. Se basant sur la solidarité intergénérationnelle, il offre l'opportunité d'échanges enrichissants. Avec I'aide et l'encadrement du Service d'aide, l'aîné et la famille d'accueil partagent un quotidien empreint de respect et de solidarité. 
Au niveau de la direction, les services ont été motivés par le fait de participer au développement de ce qui leur semblait être une des solutions au problème de la prise en charge des personnes âgées sans que ce soit trop onéreux pour les pouvoirs publics, et relativement abordable financièrement pour la personne âgée. Accessoirement, le projet permettait à la direction d'acquérir une expérience dans la gestion de projet et aussi d'améliorer l'image et la visibilité du service. Seule la mise en œuvre de l'étude du projet a pu se réaliser par l'embauche de coordinateurs permettant de réfléchir à la construction du dispositif.

Les services ont réalisé, eux-mêmes, l'information du grand public et des professionnels du projet pour susciter l'intérêt tant des personnes âgées que des familles accueillantes. Ils ont été à la rencontre des personnes âgées candidates à l'accueil familial pour évaluer leurs attentes et leur état de santé. Ils ont rencontré les familles candidates pour évaluer leurs motivations, leurs disponibilités et le niveau de formation des accueillants. Ils ont visité les maisons pour évaluer le cadre architectural déterminant les possibilités d'accueil et les conditions de sécurité.

Ce sont encore les services qui ont établi un système de financement de l'accueil acceptable pour la famille et pour les personnes âgées. Ils ont mis en relation les familles et les personnes âgées. Ils ont lancé une campagne d'information par province. Pour ce faire, ils ont effectivement engagé des coordinateurs spécialement dédiés au projet. Ils ont informé l'ensemble des travailleurs du service en réalisant des réunions expliquant le projet.

Le lien avec les familles n'a pas pu concrètement fonctionner, car certaines questions n'étaient pas réglées légalement. Par exemple, le cadre légal, fiscal et social des familles d'accueil n'était pas fixé. Beaucoup de questions concrètes restent sans réponse (exemption d'impôt pour la contribution des bénéficiaires, statut professionnel de I'accueillant, impact de ce statut sur les autres membres de la famille, déduction fiscale pour les habitations...). La problématique de domiciliation de la personne âgée accueillie s'est également posée. Faut-il la domicilier au CPAS pour maintenir la possibilité de bénéficier de la GRAPA ? ${ }^{10}$

Il est également nécessaire de créer des collaborations avec les maisons de repos pour permettre de participer à des activités en 
centre de jour. Mais ces institutions voient cet appel à projets comme une forme de concurrence. L'accueil familial ne pouvait pas toujours se réaliser dans la zone géographique fixée par la personne âgée. Certaines familles trouvaient l'indemnité proposée trop faible. L'appel à des associations disposant d'une expérience de terrain dans le domaine des familles et des aînés avait évidemment tout son sens. Néanmoins, il semble qu'il aurait été préférable que l'essentiel du cadre légal, social, et fiscal soit défini préalablement. II semble que la principale difficulté provienne du fait qu'il s'agissait d'un projet lancé par la Région wallonne, mais la modification du cadre légal dépendait, quant à lui, essentiellement du Fédéral.

En conséquence, ce projet est à l'arrêt à partir de l'année 2015.

\subsection{Maisons d'accueil communautaire (MAC)}

En 1999, un service invente, en partenariat avec une commune, le concept de maisons d'accueil communautaire.

"De nombreuses personnes âgées souffrent de solitude et/ou d'isolement social et risquent à tout moment d'être en rupture de lien social. La maison d'accueil communautaire est ouverte à toutes les personnes âgées de plus de 65 ans. (...) En général fréquentent ces maisons des personnes âgées de 70 à 85 ans, présentant différents profils (personnes autonomes, dépendantes, valides...). Les maisons d'accueil communautaire mènent une action préventive afin d'éviter que les aînés soient en rupture de lien social. La maison d'accueil communautaire propose aux aînés des activités sociales, culturelles ayant pour objectif de maintenir et/ou d'améliorer leur autonomie tant sociale que physique ${ }^{11}$.

Un appel à projets de la coopérative CERA offre l'opportunité de développer et de diffuser le concept dans le cadre de projets sociétaux. En vue de répertorier les besoins en la matière, certaines communes ou CPAS ont soumis à leurs concitoyens une enquête permettant de situer les besoins des personnes âgées de plus de 65 ans.

L'accompagnement de la coopérative CERA a permis d'embaucher une coordinatrice pendant 3 ans, de mettre en œuvre un programme de formation des animateurs, de créer 4 maisons d'accueil communautaire supplémentaires en collaboration avec d'autres communes candidates. Par cet appel à projets, le concept a été construit de manière plus élaborée. Des plaquettes d'information ont été diffusées 
durant une journée d'étude. De plus, lorsque l'accompagnement de CERA s'est terminé, le projet s'est poursuivi et les ASBL encadrant juridiquement les maisons d'accueil communautaire ont pu accéder à des financements des postes d'animateurs dans le cadre du plan Marshall dans I'aide aux personnes dépendantes (APE). Actuellement, 15 maisons d'accueil communautaire sont fonctionnelles.

Dans le cadre de cet appel à projets, l'apport du financement de CERA a permis de consolider le concept, de le diffuser et de le développer. C'est grâce à cette impulsion que le financement des postes des animateurs a été validé. C'est une reconnaissance partielle, mais les pouvoirs publics n'ont toujours pas pris le relais, en agréant ce type d'expérience et en pérennisant leur cadre législatif et financier. La mise en œuvre de ces projets pour les antennes régionales du service a renforcé leur ancrage dans un réseau local politique (commune/CPAS) et avec la population des localités concernées. En effet, beaucoup de bénévoles participent à ce projet (transport, animation d'activités...). Pour certains travailleurs des services, ils ont pu évoluer dans leur parcours professionnel: d'aide familiale vers animateur-trice MAC.

Pour les usagers dépannés par les SAFA, cette offre est complémentaire de l'aide qu'ils reçoivent à domicile tout en étant différente, car les activités des maisons d'accueil communautaire développent davantage le lien social avec une population villageoise. Pour leur entourage, les moments passés dans la maison d'accueil communautaire sont une occasion de répit qui leur permet souvent de vaquer à leurs propres activités.

\subsection{Transition du domicile à la maison de repos}

Dans le parcours de vie de la personne vieillissante, la transition du domicile vers la maison de repos est une étape importante et parfois problématique. L'objectif de l'accompagnement est d'optimaliser le passage du domicile vers la maison de repos afin d'assurer que celui-ci s'inscrive dans la continuité d'un projet personnel de " bien vieillir ". II s'agit de faire en sorte que l'entrée en maison de repos ne soit plus vécue par la personne âgée comme "une nouvelle vie ", parfois "forcée " et en rupture avec la vie à domicile, mais comme une étape cohérente dans la construction de son parcours de vie.

Dans ce cadre, les professionnels qui entourent la personne âgée 
(avant et après l'entrée en maison de repos) jouent le rôle de " Passeurs " et lui permettent de participer activement à la décision. Le développement d'une telle méthode de décision répond à une volonté de prévention. II s'agit de limiter les scénarios de " placement catastrophe ", ainsi que les placements décidés par une série d'intervenants extérieurs, sans que soit pleinement prise en compte l'opinion de la personne âgée.

Ce projet a été financé ponctuellement par une bourse de la Province du Luxembourg gérée par le conseil consultatif des aînés. Pour les aides familiales, ce projet leur a permis d'accepter plus facilement le départ des usagers vers une MR/MRS. La transition est plus harmonieuse, car elles gèrent encore quelques contacts avec la personne âgée en institution.

Pour la direction, ce programme a permis d'expérimenter la méthodologie de la gestion de projets, et de renforcer le lien avec la Province du Luxembourg.

Les travailleurs sociaux ont développé la collaboration avec le CPAS et une maison de repos. Mais ce savoir-faire est acquis, et il pourra être reproduit dans d'autres communes.

Pour I'usager, ce projet de transition renforce la qualité de l'aide reçue puisque $c^{\prime}$ est lui qui décide le départ en maison de repos ou maison de repos et de soins. II a le temps de prendre sa décision en toute quiétude et il est plus conscient de son choix. Il peut aussi expérimenter cette éventuelle forme d'hébergement. Pour pérenniser ce projet, il faudrait pouvoir stabiliser son financement. Comme dans les autres projets, c'est ce qui constitue la principale difficulté.

\section{Retour analytique transversal sur les effets des appels à projets dans l'aide à domicile}

\subsection{Nouvelles offres de service et création d'emploi}

Le secteur de l'aide à domicile a donc développé de nombreux appels à projets prouvant son caractère innovant. II est régulièrement sollicité par des promoteurs parce qu'il est un secteur avec un fort potentiel de développement actif d'emploi touchant des catégories de chômeurs peu qualifiés. Les appels à projets permettent d'innover et d'anticiper de nouvelles offres de service à la population en les expérimentant concrètement. 


\subsection{La question concomitante de la pérennisation}

Pour tous les projets commentés, la difficulté majeure est de pérenniser les appels à projets, c'est-à-dire de trouver un financement récurrent et d'obtenir que les pouvoirs publics fixent un cadre légal et règlementaire. En agissant de la sorte, ils empêchent que les expériences soient reconnues, diffusées, dupliquées. Le processus d'innovation est parfois valorisé par l'attribution de "prix " dans le cadre de concours. Cette méthodologie est contraire à la construction de trajectoires innovantes stables qui encouragent l'amélioration permanente.

Quand les appels à projets émanent des pouvoirs publiques, le défi est de les pérenniser, car souvent les projets mis en œuvre par certains vont être détricotés par ceux qui les remplacent (surtout s'ils ne sont pas de la même étiquette politique).

Quand des appels à projets émanent de fondations ou de coopératives, le programme de suivi des promoteurs est plus intensif. Mais quand il se termine, il est rarement pérennisé dans un cadre légal.

Beaucoup d'appels à projets sont lancés sans que les décideurs politiques examinent toutes les conséquences de leur mise en œuvre. Ils doivent parfois s'interrompre en attente d'un cadre légal à définir.

Ces incertitudes sur le caractère pérenne entrainent une précarisation de l'emploi non négligeable.

\subsection{La face cachée du projet : réflexivité et diversification}

Pourtant, mener des projets innovants, met l'association en réflexion sur elle-même. Elle peut revisiter son offre de services à la population et dessiner les voies du futur. Elle peut développer de nouveaux métiers ou de nouvelles méthodologies de travail et permettre les évolutions professionnelles. La façon même dont l'organisation, le service vont se saisir de l'instrument orientera les prises de décisions et donc sa réflexion liée au projet. Elle peut aider à réviser les concepts de l'aide à domicile, développer le travail en réseau, améliorer la communication et la gouvernance.

A partir des projets décrits, les services d'aide à domicile ont entamé des stratégies de diversification, car cela leur permet de se distinguer de la concurrence, de mieux s'ancrer dans un travail en réseau local, d'affirmer leur savoir-faire professionnel face au travail 
au noir ou au bénévolat, d'assurer une couverture géographique plus complète. Ils ont su également faire évoluer leurs conceptions de l'aide à domicile.

\subsection{La face visible: innovation et parole aux usagers}

Plusieurs projets tendent à faire évoluer la notion de domicile (accueil familial) ou d'alternatives à une vie complète à domicile (maisons d'accueil communautaires) ou de transition entre une vie à domicile et une prise en charge institutionnelle. Ils ont aussi veillé à intégrer la problématique des aidants proches en leur offrant des formes de répits et de conseils, en sécurisant leur habitation et en l'adaptant aux évolutions de santé et/ou de handicap de la personne aidée. Ils ont également veillé à diminuer l'isolement des aînés en proposant des actions de lien social (maisons d'accueil communautaire) avec des professionnels et des bénévoles ou en adaptant leurs politiques de travail pour étendre leurs activités hors du domicile de I'usager (GAD). Ils ont aussi défendu le principe de l'accessibilité des services aux usagers en établissant une barémisation expérimentale des activités de GAD et de GEM.

Ils ont permis d'écouter la parole des usagers soit en leur permettant de mener des actions d'évaluation (projet communication), soit en confortant le libre-choix de vie par les usagers (transition domicile /MR-MRS ou accueil familial). En cela, ces services anticipent les contours de ce qui devra guider la réflexion des politiques sociales futures: " dessiner les trajectoires de vie des personnes et leur permettre de continuer à vivre une vie de qualité en favorisant les transitions de leur prise en charge ".

\subsection{Organisation et conditions de travail}

Ces projets ont aussi eu beaucoup d'impact pour les travailleurs des services. De nouveaux métiers ont été créés ou consolidés (GAD, GEM, OP, ergothérapeutes, coordinateurs, animateurs, MAC...) permettant l'insertion socioprofessionnelle de demandeurs d'emploi peu qualifiés ou qualifiés.

De nouveaux programmes de formation se sont développés accentuant la spécialisation de certains professionnels (projet répit pour personne handicapée) et profitant finalement à l'ensemble des prestataires du service. Ces projets contribuent également à faire 
évoluer la répartition des tâches entre les différents métiers. Cette évolution est nécessaire, car la pénurie de certains profils professionnels et la croissance des besoins demandent des glissements d'actes entre le secteur sanitaire et le secteur social.

A travers la diversification des métiers, on voit également se construire des parcours professionnels. Une AF devient animatrice de MAC. Un assistant social devient coordinateur du projet d'accueil familial. C'est une grande richesse pour leur secteur de pouvoir permettre à un travailleur d'utiliser son expérience professionnelle dans un autre lieu ou avec un autre public. C'est une réelle source de réalisation personnelle pour les travailleurs impliqués.

La principale valeur des appels à projets est de mettre en réflexion les acteurs des associations, de les amener à évaluer leurs actions, à innover sur le terrain dans leur méthodologie et dans leur finalité en tenant compte des nouvelles demandes des usagers. Ils permettent de spécialiser certains travailleurs dans le suivi de certains groupes de bénéficiaires. Ce focus est souvent l'occasion d'approfondir avec eux la réponse aux demandes qu'ils formulent. Ces temps dédiés sont souvent gages d'élaboration de critères d'un accompagnement de qualité et se répercute sur l'ensemble des métiers de prestataires. Le développement des appels à projets constitue une pépinière de type d'actions à déployer, pour autant que les pouvoirs publics collationnent ce savoir-faire, l'évaluent, le structurent, le perpétuent et le diffusent.

\section{Notes}

(1) Pour rappel, il existe quatre régimes différents sur le territoire belge. Voir à ce propos: Dumont G-H. (2011).

(2) La Direction Générale opérationnelle des Pouvoirs locaux, de l'action sociale et de la santé est communément appelée DGO5.

(3) Nous renvoyons le lecteur aux rapports annuels de la Banque Nationale de Belgique sur le poids des institutions sans but lucratif, ainsi qu'à Artois et Hamzaoui (2013).

(4) Voir Guéguen (2011).

(5) Voir à ce propos Accaputo, Bayen, Pagano (2006).

(6) Ces mesures d'aide à l'emploi sont reprises sous l'acronyme APE et consistent à octroyer, sous forme de points, une aide annuelle visant à 
subsidier partiellement la rémunération des travailleurs, une réduction importante des cotisations patronales de sécurité sociale. Cette aide est accordée afin de favoriser l'engagement de demandeurs d'emploi inoccupés de longue durée.

(7) Extrait de la classification professionnelle sectorielle: CCT de la SCP 318.01 du 19 mars 2009 fixant les conditions de travail, de rémunération et d'indexation pour le personnel des SAFA en région wallonne.

(8) La carrière d'une GAD s'échelonne sur 29 ans dans le cadre de la grille barémique de la SCP 318.01.

(9) Une heure inconfortable est une heure prestée entre $6 \mathrm{~h} 00$ et $8 \mathrm{~h} 00$ et entre $18 \mathrm{~h} 00$ et $21 \mathrm{~h} 30$, ou effectuée les weekends et les jours fériés. Ces heures génèrent un supplément de subvention de la Région wallonne.

(10) GRAPA : la garantie de revenus aux personnes âgées est une prestation octroyée aux personnes âgées dont les revenus sont trop faibles pour assurer leur subsistance. Elle remplace l'ancien « revenu garanti » et s'obtient en référence à des conditions d'âge, de nationalité et de résidence.

(11) ADMR (1999).

\section{Bibliographie}

Accaputo A., Bayen B., Pagano C., 2006, « Le Plan Marshall pour la Wallonie », Courrier hebdomadaire du CRISP, 1919-1920.

ADMR, 1999, Qu'est-ce que les MAC?, Brochure d'automne.

ARTois P., HamzaOui M. (dir), 2013, Reconfigurations de l'associatif: salariat et reconnaissance du travail, Les Politiques Sociales, vol $3 \& 4$.

Dumont G-H., 2011, La Belgique, hier et aujourd'hui, Paris, Presses Universitaires de France.

GuÉguen J-Y., 2011, L'année de l'action sociale 2011 - Social et médicosocial: les nouvelles règles du jeu, Paris, Dunod. 